\title{
EchoGéo
}

$49 \mid 2019$

Les corridors de développement

\section{Corridors et circulations au temps de la mondialisation}

Jean-Louis Chaléard

\section{OpenEdition}

1 Journals

Édition électronique

URL : https://journals.openedition.org/echogeo/18156

DOI : $10.4000 /$ echogeo. 18156

ISSN : 1963-1197

Éditeur

Pôle de recherche pour l'organisation et la diffusion de l'information géographique (CNRS UMR 8586)

Référence électronique

Jean-Louis Chaléard, "Corridors et circulations au temps de la mondialisation », EchoGéo [En ligne], 49 | 2019, mis en ligne le 25 octobre 2019, consulté le 11 août 2021. URL : http://

journals.openedition.org/echogeo/18156 ; DOI : https://doi.org/10.4000/echogeo.18156

Ce document a été généré automatiquement le 11 août 2021.

EchoGéo est mis à disposition selon les termes de la licence Creative Commons Attribution - Pas d'Utilisation Commerciale - Pas de Modification 4.0 International (CC BY-NC-ND) 


\title{
Corridors et circulations au temps de la mondialisation
}

\author{
Jean-Louis Chaléard
}

1 Les corridors de développement ont été l'objet de beaucoup de discussions et de projets, d'ampleur variable, parfois pharaoniques. Ils ont été promus par l'ONU et les institutions financières internationales qui les ont favorisés et ont participé à leur mise en œuvre, en collaboration avec les États et de grands opérateurs économiques privés. Leur existence renvoie à de nombreuses questions qui touchent au développement des territoires, à l'organisation de l'espace et qui préoccupent les aménageurs mais aussi les chercheurs en sciences sociales, particulièrement les géographes. La présente livraison d'Échogéo leur est largement consacrée, ainsi qu'à d'autres circulations dans le contexte actuel de mondialisation.

2 Le dossier de la rubrique Sur le champ, coordonné par N. Fau, porte sur « Les corridors : de nouveaux outils de développement? » Il existe de nombreuses études, provenant des chercheurs, des organisations et des institutions financières internationales sur les corridors, leur mise en place, leurs conséquences en termes de développement économique. Face à cette abondante littérature, N. Fau, dans son introduction, souligne l'importance d'une approche critique de la notion de corridor. Et l'intérêt du dossier réside dans ses interrogations sur les effets structurants et les formes de territorialisation induits par ces grandes infrastructures. Le corridor doit non seulement relier les villes entre elles par des axes de communication performants, mais développer un nouveau type d'espace multipolaire et transnational, articulant entre elles des régions urbaines existantes et des régions émergentes. Qu'en est-il dans la réalité?

3 Comme le relève N. Fau, la notion de corridor s'étend aujourd'hui au monde entier. Les sept articles du dossier le confirment : ils présentent une grande variété géographique, puisque on y rencontre des cas pris sur la plupart des continents, de l'Amérique centrale à l'Asie du sud-est en passant par la Sibérie, l'Europe occidentale, l'Afrique orientale, l'Inde, etc. Les façons d'aborder l'objet diffèrent aussi. Certains articles traitent de corridors sur une grande partie de leur étendue (comme A. Cariou pour le 
Transsibérien), sur un éventail de corridors voisins et concurrents (comme ceux d'Amérique centrale analysés par L. Medina), sur une partie spécifique (ainsi le corridor entre Rhin et Rhône, inscrit dans la liaison Méditerranée-mer du Nord, étudié par G. Carrouet) ou insistent sur une métropole au cœur du corridor: Nairobi pour le Northern Corridor est-africain (V. Fourault-Cauët et J.-F. Steck), Chiang Raï pour le corridor du Grand Mékong (T. Foin). Pour P. Cadène et Y.-M. Rault, le cadre d'étude est large puisqu'il s'agit d'analyser les corridors industriels en s'interrogeant sur les stratégies de développement régional de l'État indien et d'implantation des grandes firmes privées.

4 Au-delà de cette diversité, quelques interrogations ou lignes de force se dégagent d'un article à l'autre. Axe de transport, espace polarisant au niveau régional, la définition du corridor et de son aire d'influence sont des interrogations que l'on retrouve dans pratiquement tous les textes. À propos de Nairobi les auteurs montrent l'existence de corridors de nature différente, un corridor écologique s'opposant au corridor de développement économique. L. Medina analyse plusieurs corridors dont la philosophie, comme la mise en place et les moyens mis en œuvre, varient selon les États en Amérique centrale. N. Fau montre les différences entre les types de corridors, terrestres ou maritimes, entre autres. La plupart des contributions s'interrogent sur leur organisation interne, leur rôle dans les intégrations régionales, les différenciations spatiales dont ils sont porteurs ou pas : ainsi, P. Cadène et Y.-M. Rault replacent-ils les corridors indiens dans les cadres régionaux qu'ils contribuent à construire. Les textes insistent sur la dimension économique certes, mais aussi sur les dimensions sociale et géopolitique comme le marquent fortement l'exemple du corridor eurasiatique (A. Cariou), celui de l'Amérique centrale (L. Medina), qui voit se multiplier les projets, avec des initiatives étatiques fragmentées. Les enjeux géopolitiques sont au cœur des entreprises, que ce soit à l'échelle internationale (les corridors de la Région du Grand Mékong) ou nationale (les corridors industriels indiens), entraînant souvent des rivalités entre État (comme en Amérique centrale). L'analyse débouche aussi fréquemment sur la question de la gouvernance, qu'elle soit à l'échelle nationale (pour penser la gestion du territoire) ou internationale (puisque la plupart des corridors nécessitent des accords entre les différents pays concernés par les infrastructures mises en place), comme le met particulièrement en évidence l'étude du corridor RhinRhône.

5 Les auteurs s'interrogent sur les conséquences en termes de développement et d'organisation de l'espace des corridors. Leurs effets sont d'importance variable et pas toujours positifs. Le Transibérien, vu par A. Cariou, ne joue son rôle de corridor qu'aux extrémités, car les territoires traversés sont utilisés comme supports ou comme périphéries pourvoyeuses de matières premières. En Amérique centrale, L. Medina montre que les nombreux corridors ont, pour l'instant, absorbé beaucoup d'énergies pour peu de résultats. V. Fourault-Cauët et J.-F. Steck, s'intéressant aux effets d'un corridor à l'échelle locale, avec l'exemple de Nairobi, montrent que le tracé du corridor destiné à désenclaver l'intérieur du Kenya et la région des Grands Lacs interfère avec les objectifs de conservation de la nature qui lui sont attribués. Ils relèvent les stratégies contradictoires des acteurs autour de la place de corridors de différentes natures: de développement pour l'un, écologique pour l'autre. N. Fau, à partir de l'exemple de l'Asie du sud-est, s'interroge sur la façon d'étudier les corridors de développement. Ceux-ci, promus par la Banque asiatique de développement à la fin des années 1990, ont donné lieu à une abondante littérature. C'est l'occasion pour elle de 
les présenter dans leur diversité et de s'interroger sur la meilleure façon d'aborder leur bilan. Alors que les institutions internationales voient en eux des outils d'intégration économique, les chercheurs déconstruisent les discours opérationnels, mettant en évidence les enjeux de pouvoirs sous-jacents aux créations d'infrastructures de transport et les écarts entre discours et réalité. P. Cadène et Y.-M. Rault soulignent que les corridors planifiés par l'État indien ne sont pas tous accompagnés par les investisseurs, qui privilégient d'autres corridors industriels et, surtout, les grandes métropoles accentuant ainsi leur domination économique. T. Foin montre les effets différenciés sur les villes de part et d'autre des frontières, dans le cas de la Région du Grand Mékong, et relève que le processus de régionalisation généré par les corridors peut induire une reconfiguration urbaine des villes. G. Carrouet analyse la perspective $\mathrm{du}$ corridor de développement Rhin-Rhône, comprenant un ensemble de villes moyennes de Dijon à Bâle pour lesquelles l'arrivée de la grande vitesse ferroviaire a été perçue comme un facteur favorable à la constitution d'un réseau de villes moyennes, mais dont le bilan est décevant au regard des ambitions de départ. Au total, si les contributions sur l'Asie du sud-est et l'Europe occidentale mettent en évidence l'émergence de nouvelles polarités, plus que les autres, presque toutes soulignent les difficultés en termes de gestion et l'absence de prise en compte des questions environnementales.

6 La rubrique Sur l'écrit aborde aussi des questions de développement et de mobilité dans le contexte de mondialisation. Mais le sujet, comme l'approche, fournissent un contrepoint et un complément au dossier sur les corridors. J.-F. Steck interview A. Choplin et $\mathrm{O}$. Pliez à propos de leur livre La mondialisation des pauvres publié en 2018. Partant de lieux éparpillés dans le monde arabe, au cœur de la Méditerranée ou en Afrique subsaharienne, et analysant des filières de produits et d'objets, les auteurs mettent en évidence de nouvelles circulations, apparues depuis une trentaine d'année, loin des succès des multinationales mais qui concernent plusieurs milliards d'individus et qui relient la Chine et l'Asie du sud-est au reste du monde, notamment l'Afrique.

7 Les migrations internationales, les flux illégaux et illicites, le commerce informel s'inscrivent dans des spatialités parfois très différentes des corridors de développement. Ce que l'on a pu qualifier de «mondialisation par le bas " invite à questionner l'existence et le rôle structurant de routes commerciales transnationales indépendantes des stratégies institutionnelles. Mais ses acteurs restent pauvres pour la majorité d'entre eux. En ce sens, le livre de A. Choplin et 0 . Pliez ne prétend pas offrir une solution pour le développement, mais l'interview des auteurs autorise une mise en perspective du dossier sur les corridors. 